\title{
A role for short days in sustaining seasonal reproductive activity in the ewe*
}

\author{
B. Malpaux† and F. J. Karsch \\ Reproductive Sciences Program and Department of Physiology, The University of Michigan, \\ Ann Arbor, MI 48109-0404 USA
}

\begin{abstract}
Summary. This study tested the hypothesis that short days can prolong the breeding season of the ewe when reproductive activity is initiated by an endogenous process, as opposed to when it is driven by exposure to short days. Suffolk ewes were ovariectomized and treated with Silastic capsules containing oestradiol; reproductive activity was monitored from serum concentrations of LH. In this model, a rise in LH is indicative of onset of the breeding season and the duration of the elevation in LH is an indicator of length of reproductive activity. The ewes were subjected to 6-month alternations between long and short photoperiods such that the $\mathrm{LH}$ rise began during exposure to the inhibitory long photoperiod (i.e. it resulted from an endogenous process). When short days were provided soon after such a spontaneous onset of the LH rise, the duration of reproductive activity was greater than that observed when ewes were maintained in long days throughout the LH elevation (194 \pm 10 vs $155 \pm 15$ days; $P<0.02$ ). Since the transition from anoestrus to the breeding season in Suffolk ewes maintained outdoors does not require a decrease in daylength and appears to be generated by an endogenous process, our results support the hypothesis that shortening photoperiod sustains the natural breeding season which begins in early autumn.
\end{abstract}

Keywords: seasonal reproduction; photoperiod; photorefractoriness; LH; breeding season; sheep

\section{Introduction}

Because the breeding season of the ewe occurs during the autumn and early winter, the sheep has been classified as a 'short-day breeder'. This classification implies that short days are somehow inductive to the reproductive process in this species. Nevertheless, there is an increasing body of evidence that reproductive onset in the autumn does not require a decrease in photoperiod (Robinson et al., 1985b; Worthy et al., 1985; Jackson et al., 1988). Rather, exposure to long days in the spring and summer appears to synchronize an endogenous process that leads to a spontaneous initiation of breeding activity in the autumn (Malpaux et al., 1989; Wayne et al., 1990). Recent findings suggest that one role for short days is to maintain the breeding season for its full duration (Malpaux et al., 1988b; O'Callaghan et al., 1989a; Wayne et al., 1990). Tests of the hypothesis that short days sustain reproductive activity have utilized various photoperiod manipulations and, in all of these, reproductive onset was driven by a reduction in daylength. Since the natural breeding season appears to be initiated by an endogenous process rather than by exposure to short days (Robinson et al., 1985b; Malpaux et al., 1989; Karsch et al., 1989), the question arises as to whether short days can prolong a reproductive season that is generated endogenously. In the present study,

\footnotetext{
*Reprint requests to Fred J. Karsch.

†Present address: INRA Station de Physiologie de la Reproduction, Centre de Recherches de Tours, 37380 Nouzilly, France.
} 
we tested the hypothesis that short days can extend the breeding season of Suffolk ewes when the reduction in daylength is provided after an endogenously generated onset of reproductive activity. In this report, we consider a reproductive period which begins spontaneously during prolonged exposure to an inhibitory long photoperiod to have been generated by an endogenous process.

\section{Materials and Methods}

\section{General}

The study was performed on sexually mature Suffolk ewes maintained in a light-sealed room at the Sheep Research Facility in Ann Arbor, Michigan ( $42^{\circ} 18^{\prime} \mathrm{N}$ latitude). Daylength was regulated by an electronic timer that operated fluorescent bulbs providing approximately 350 lux at eye level. Photoperiod was either $16 \mathrm{~h}$ light: $8 \mathrm{~h}$ dark, 16L:8D (hereafter referred to as long days) or $8 \mathrm{~L}: 16 \mathrm{D}$ (short days) with lights on at $06: 00 \mathrm{~h}$ in both instances. A dim red light ( $<3$ lux) remained on continuously to facilitate night-time collection of blood for another study (Malpaux et al., 1988a). Ewes were fed hay daily and had free access to mineral licks and water. At least 3 months before the study, each ewe was ovariectomized and given a subcutaneous Silastic capsule containing a $30-\mathrm{mm}$ column of oestradiol- $17 \beta$ to maintain a physiological serum oestradiol concentration of $3-5 \mathrm{pg} / \mathrm{ml}$ (Legan et al., 1977; Legan \& Karsch, 1980). The capsules were replaced once a year to ensure that they did not become depleted of oestradiol. Reproductive neuroendocrine activity was assessed from the concentration of serum luteinizing hormone (LH) in blood samples taken twice weekly. Periods of elevated LH are indicative of the breeding season; low LH values signify anoestrus (Legan et al., 1977; Legan \& Karsch, 1980). These changes in LH secretion are photoperiodically mediated and reflect a shift in response to oestradiol negative feedback which constitutes a primary neuroendocrine mechanism for seasonal changes in ovarian cyclicity (Legan \& Karsch, 1979, 1980).

\section{Experimental design}

The experiment was conducted in two phases spanning a period totalling $3 \frac{3}{4}$ years during which photoperiod was alternated between long and short days as indicated in Fig. 1. The first phase was performed on 3 ewes to test the feasibility of the experimental approach and to gather initial results on the role of short days. Two ewes were added in the second phase to increase group size for further testing the role of short days.

Phase 1 compared the duration of reproductive induction (LH elevation) under two conditions: (1) when the LH rise was driven by transfer from long to short days; (2) when short days were provided after LH had increased spontaneously in long days. Three ewes were treated with 70 long days beginning in February (early anoestrous season) to establish reproductive neuroendocrine suppression (a, in Fig. 1). Photoperiod was then switched to short days (24 April 1984) to drive an LH rise and thus determine duration of the reproductive period induced by transfer from long to short days (b, in Fig. 1; LH Rise I). After 184 short days, photoperiod was switched back to long days (c, in Fig. 1). After 196 days of exposure to long days, LH had increased spontaneously. Photoperiod was then switched back to short days for 173 days to determine the duration of the LH rise when short days were provided after an endogenously generated reproductive onset under long days (d, in Fig. 1; LH Rise II). Phase 1 of the study ended after this 6-month exposure to short days.

Phase 2 compared the duration of the LH elevation under two conditions: (1) when short days were provided just after a spontaneous reproductive onset in long days; (2) when short days were not provided at all. Upon completion of Phase 1 ( 29 October 1985), 2 ewes which had been primed with 70 short days were added to the study. At the beginning of Phase 2, photoperiod was switched back to long days which were maintained until just after LH in each individual was determined to have increased (e, in Fig. 1). Photoperiod was then switched back to short days ( $f$, in Fig. 1; actual time of transfer was determined for individual ewes by serum LH values and averaged $26 \pm 3$ days after LH had started to increase; exposure to long days averaged $204 \pm 10$ days). This part of the study determined the duration of the LH elevation when ewes were exposed to short days just after reproductive onset (Fig. 1, LH Rise III). Once LH had fallen back to low levels (29 November 1986), photoperiod was switched back to long days until the end of the study 1 year later (g, in Fig. 1). The LH rise that occurred during this final exposure to long days served to determine the duration of reproductive induction in the absence of any short days (Fig. 1, LH Rise IV).

\section{Blood sampling and assays}

Blood $(5 \mathrm{ml})$ was sampled by jugular venepuncture and serum was obtained as described previously (Karsch \& Foster, 1975). LH was assayed in duplicate $25-200 \mu \mathrm{l}$ samples of serum using a procedure previously described (Niswender et al., 1968, 1969; Hauger et al., 1977). Sensitivity was $0.27 \pm 0.02 \mathrm{ng} \mathrm{NIH-LH-S12/ml} \mathrm{for} 200 \mu \mathrm{l}(2$ standard deviations from buffer control; mean \pm s.e.m., 36 assays). Intra-assay coefficient of variation (CV) for 3 serum pools determined 6 times in each assay averaged $6.8 \%$; interassay $\mathrm{CV}$ for these same pools averaged $12 \cdot 3 \%$. 


\section{Data analysis}

Rises and declines in LH concentrations were determined for each ewe by an algorithm which uses regression-like statistics with a moving variable-length window to detect changes in hormone levels (Malpaux et al., 1988b; Brown et al., 1988). This algorithm can identify 4 stages of a cycle: baseline, rise, plateau and decline. Durations of LH elevations were calculated as the interval from onset of the rise stage to the end of the subsequent decline stage. Amplitude of the LH elevation was calculated as the mean concentration during the plateau stage of the cycle. Two-tailed paired $t$ tests were used for comparison between treatments.

\section{Results}

The entire LH profile for one representative ewe during both phases of the study is illustrated in Fig. 2. Data for all ewes are summarized in Figs 3 and 4 for Phases 1 and 2, respectively. To facilitate data presentation, the 4 successive $\mathrm{LH}$ rises during the study are identified in text and figures as LH Rise I, II, III and IV (see Fig. 1, design).

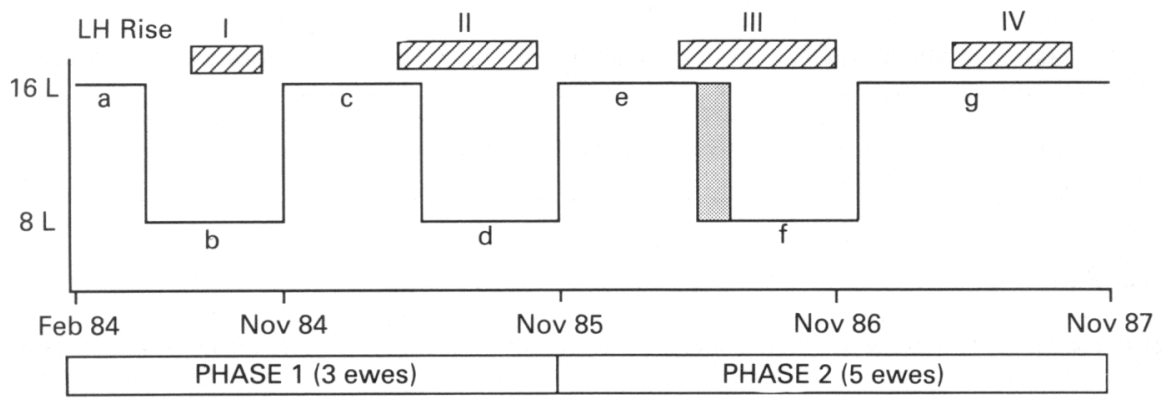

Fig. 1. Experimental design. Photoperiod is indicated on vertical axis; $16 \mathrm{~L}$ signifies long days of $16 \mathrm{~L}: 8 \mathrm{D}$ and $8 \mathrm{~L}$ indicates short days of $8 \mathrm{~L}: 16 \mathrm{D}$. Time and phase of experiment are indicated along horizontal axis. Sequential photoperiodic treatments are identified as a-g and successive LH rises as I-IV (shaded boxes). The shaded area between e and $f$ represents the interval during which the ewes were transferred to short days about 25 days after $\mathrm{LH}$ concentrations increased. See 'Materials and Methods' for detail.

\section{Phase 1}

LH Rise I began while ewes were exposed to short days whereas LH Rise II began the following year while ewes were still being exposed to long days (Fig. 2). In each of the 3 ewes, LH Rise I was shorter than LH Rise II ( $84 \pm 10$ vs $158 \pm 31$ days; mean \pm s.e.m.; Figs 2 and 3 ). There was no difference in amplitude of these two LH elevations.

\section{Phase 2}

The photoperiodic conditions for LH Rise III differed from those for Rise IV in that ewes were transferred to short days soon after the onset of Rise III but no short days were provided during LH Rise IV (Fig. 2). There was no difference in the time LH started to increase relative to the first long day in these two instances (after $179 \pm 9$ long days for Rise III; $168 \pm 9$ long days for Rise IV). In each ewe, however, LH remained elevated longer in the presence of short days than in their absence $(194 \pm 10 \mathrm{vs}$ $155 \pm 15$ days; $P<0.02$; Figs 2 and 4). Further, amplitude of the $\mathrm{LH}$ plateau was greater in the presence of short days than in their absence $(7 \cdot 1 \pm 0.4$ vs $5.4 \pm 0.6 \mathrm{ng} / \mathrm{ml} ; P<0.05)$. 


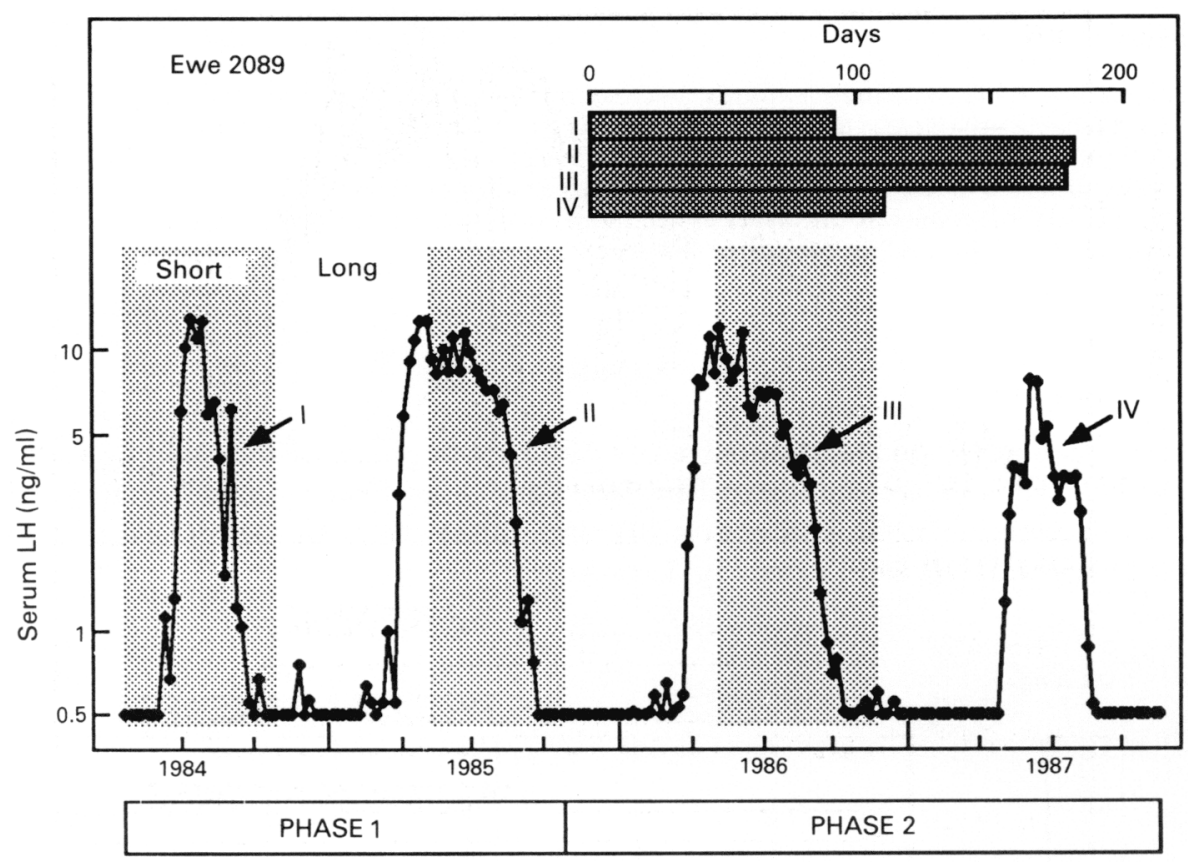

Fig. 2. Serum LH concentrations in a representative ewe throughout the entire experimental period (Phases 1 and 2). Successive LH rises are depicted as I-IV. Photoperiod was alternated between short and long days as indicated by the shaded and open areas, respectively. Horizontal bars at top indicate duration of LH Rises I-IV. See Fig. 1 for design details.

\section{Discussion}

Our findings indicate that a decrease in daylength can sustain reproductive neuroendocrine activity when this activity begins in Suffolk ewes during prolonged exposure to an inhibitory long photoperiod. Since a reproductive period which begins under a long photoperiod is considered to be endogenously generated, our findings lead to the conclusion that short days can prolong the breeding season that begins as the result of an endogenous process.

Recent studies of ewes indicate that short days can sustain a reproductive period induced by an abrupt decrease in daylength. Specifically, when reproductive induction is driven by a single-step decrease in photoperiod, its duration is only 2-3 months which is far shorter than the 5-6-month breeding season observed outdoors (Thimonier et al., 1978; Karsch et al., 1986; Poulton et al., 1987; confirmed by LH Rise I in the present study). This duration can be increased by a second step-wise decrement in photoperiod before the time of reproductive onset, although the breeding period is still not as long as that of ewes outdoors (Malpaux et al,, 1988b; O'Callaghan et al., 1989a). Our present finding that short days can also sustain a reproductive season that is initiated endogenously is pertinent to the timing of reproductive activity under natural photoperiodic conditions. Specifically, the breeding season of the Suffolk ewe appears not to be driven by short days in the late summer and autumn; rather it is generated by an endogenous process that is synchronized by exposure to long days during the previous spring and summer (Robinson et al., 1985b; Malpaux et al., 1989; Karsch et al., 1989; Wayne et al., 1990). Our findings therefore support the hypothesis (Malpaux et al., 1988a) that one role for the decreasing photoperiod after the summer solstice is to maintain the natural breeding season for its full duration.

One aspect of our study, however, might seem incongruent with this hypothesis. Specifically, when no short days were given, the duration of the endogenously generated LH elevation (Rise IV) 


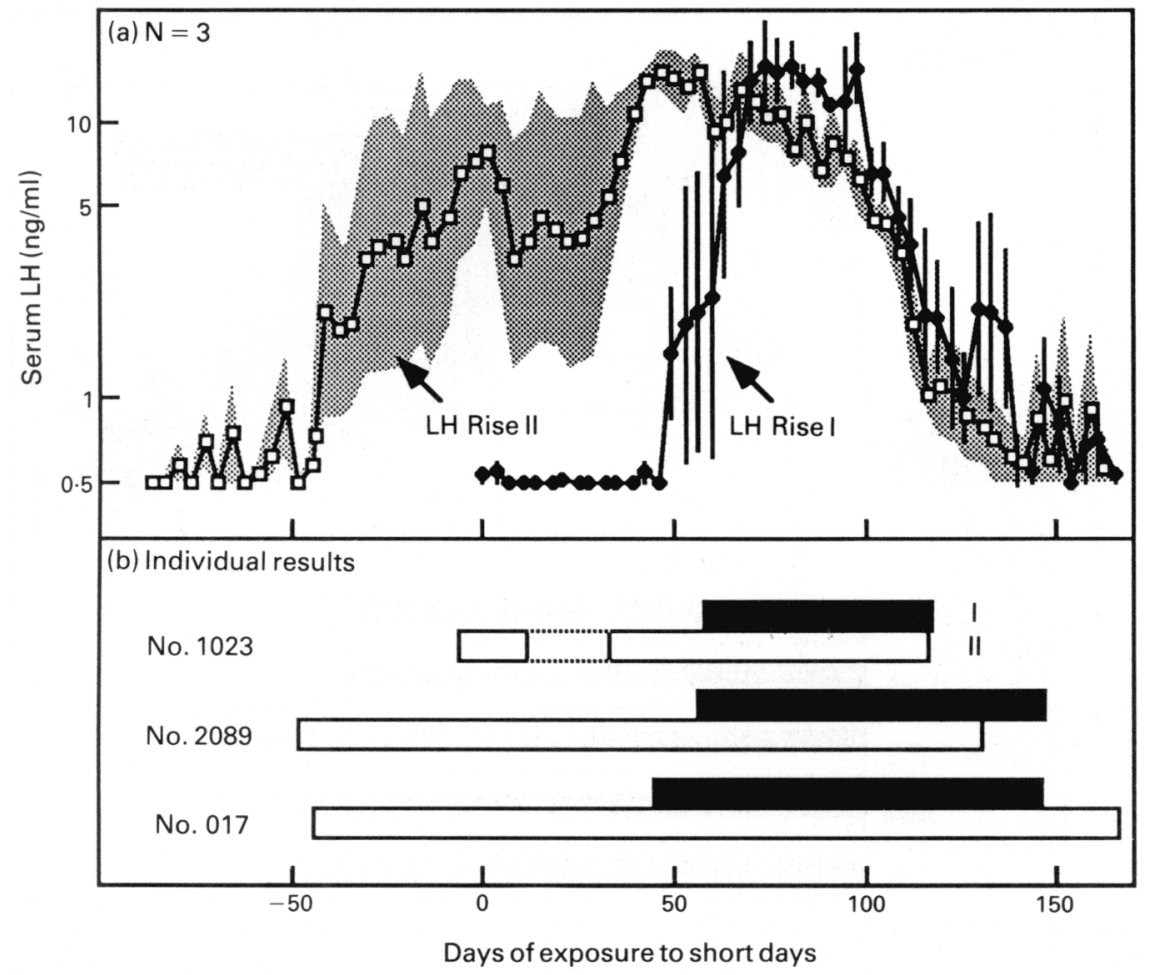

Fig. 3. Results for Phase 1 of the study. (a) Mean \pm s.e.m. (logarithmic scale) serum LH concentrations in 3 ewes exposed to a shift from long to short photoperiod on Day 0 in 2 successive years. The LH rise of the first year (I) was induced by transfer to short days; the LH rise of the second year (II) began while ewes were still being exposed to long days. (b) Bars depict duration of LH rises in each of the 3 ewes included in (a). Solid bars depict LH Rise I and the open bars signify LH Rise II. The dotted portion of LH Rise II in Ewe 1023 indicates the occurrence of a transient fall in LH soon after the LH elevation had begun.

was not shorter than that observed for ewes kept outdoors (5-6 months; Karsch et al., 1989). A similar result was described by Jackson et al. (1988) who reported that the reproductive period was not shortened by moving ewes indoors on the summer solstice and maintaining them in that long photoperiod throughout the ensuing breeding season. These findings, however, must be interpreted in view of evidence that photoperiodic history is crucial in determining the ewe's response to a given daylength (Robinson \& Karsch, 1987). We have found that exposure to a decrease in daylength makes the ewe dependent upon continued exposure to short days if reproductive activity is to be maintained (O'Callaghan et al., 1989b). Thus, once daylength begins to decrease after the summer solstice, the ewe may become committed to the shortening photoperiod to sustain the breeding season. This dependency upon shortening days is likely to dissipate by the time of the autumnal equinox, because disruption of the photoperiodic response by pinealectomy around that equinox was found to have little, if any, effect on duration of the breeding season (Wayne et al., 1990).

A potential limitation to our interpretation that short days can prolong the breeding season relates to the design of our study and the sequence of treatments. Specifically, each animal served as its own control and received the treatments in the same order. It could be argued, therefore, that our results were biased by time of year, age of the animals or photoperiodic history. Although we cannot discount these possibilities, we find them unlikely for several reasons. First, in Phase 2 of the study, in which the short-day effect was identified, the transfer to long days was performed at the same time in the autumn of two successive years to minimize any influence of non-photoperiodic 


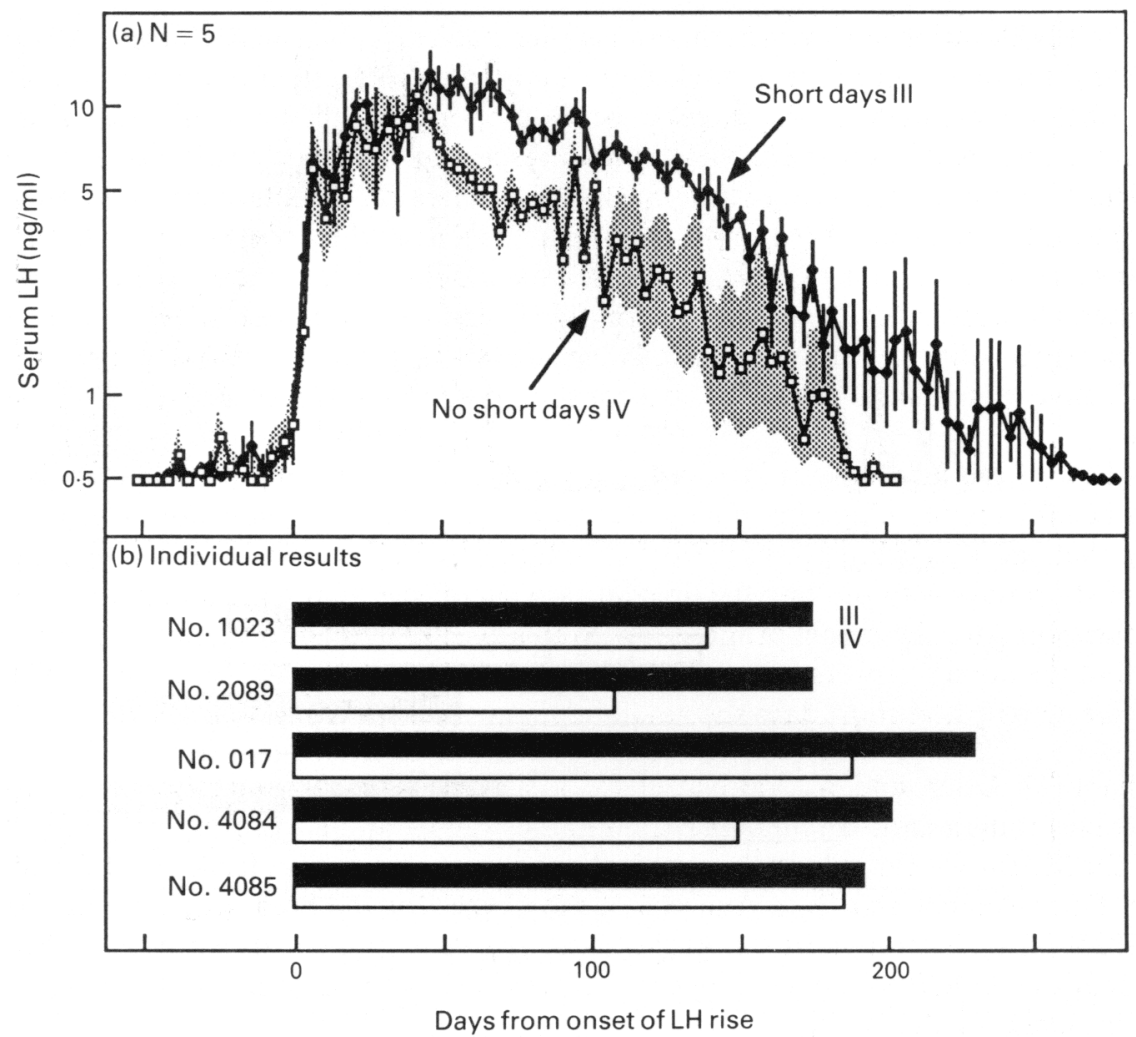

Fig. 4. Results for Phase II of the study. (a) Mean \pm s.e.m. (logarithmic scale) serum LH concentrations in 5 ewes exhibiting LH rises in 2 successive years, initially in the presence of short days (LH Rise III), and then in the absence of short days (LH Rise IV). (b) Bars depict duration of LH rises in each of the 5 ewes when short days were provided (III, solid bars) or when short days were not provided (IV, open bars).

variables and of phase of the endogenous rhythm. There was no difference in the time of onset of the LH rises in the spring (Rises III and IV); yet the durations were different. Second, 3 of the ewes received similar light treatments in 2 successive years; they exhibited essentially the same LH response in each of the 2 years (e.g., Fig. 2, LH Rises II and III). Third, although our ewes became older as the study progressed, it is unlikely that age was a factor in length of the LH elevation. All ewes were in good health at the end, and an influence of increasing age on duration of the reproductive season was not observed in Suffolk ewes which remain healthy (Karsch et al., 1989). Fourth, as concerns photoperiodic history, there was essentially no difference in duration of exposure to long days before LH Rises III and IV in Phase 2 of the study. Yet, the LH rise observed when short days were provided soon after LH had increased (LH Rise III) was prolonged relative to that when short days were not provided at all (LH Rise IV). Therefore, should photoperiodic history prove to be important in this regard, it would not discount our conclusion that short days can sustain a reproductive season that begins as the result of an endogenous process. It would appear justified, therefore, to conclude that the observed differences in duration of the $\mathrm{LH}$ elevation reflect a short-day effect and not experimental artefact.

An interesting question related to the short-day effect concerns the mechanism by which short days sustain reproductive activity. Specifically, do they provide a stimulatory signal to the neuroendocrine axis or do they reset the endogenous rhythm that underlies seasonal reproduction in the ewe? The effect probably includes at least some stimulatory action, given the ability of short days to 
heighten the LH elevation as shown in this and earlier studies (Robinson \& Karsch, 1987; Malpaux et al., 1988b). Further, a decrease in daylength can drive an increase in frequency of pulsatile $\mathrm{LH}$ release, with the magnitude of the increase being proportional to the magnitude of the photoperiodic reduction (Robinson et al., 1985a). With respect to the other possibility, it is not known whether short days also reset the rhythm. Such a determination would require a long-term study. For example, if the delay in offset of reproductive activity caused by short days could be shown to persist for successive reproductive transitions in a fixed photoperiod, then short days would have phase-shifted the seasonal cycle and thus the rhythm would have been reset. Such a study, however, might be extremely difficult to interpret given the relatively large variability in the timing of successive reproductive transitions in ewes kept in a constant photoperiod (Karsch et al., 1989; Jackson et al., 1990).

Regardless of the mechanisms involved, the main thrust of this report is that short days can provide a cue for maintaining reproductive activity in the ewe. In conjunction with other studies indicating that short days are not necessary to induce breeding activity, our findings suggest that one role of short days is to prolong the breeding season of ewes maintained in natural conditions. This is consistent with the model proposed by Malpaux et al. (1989) to explain the photoperiodic regulation of the annual reproductive cycle of the ewe, i.e. that long days synchronize the onset of reproductive activity and short days sustain it.

We thank D. Doop and K. VanNatter for assistance with the animal experimentation; B. Glover for performing radioimmunoassays; Dr G. Niswender and Dr L. Reichert, Jr for supplying reagents; Dr M. Brown for help with statistical analyses; and the core facilities of Standards and Reagents, Farm Animal and Data Analysis of the Center for the Study of Reproduction (NIHP30-HD18258). Funds to support this project were supplied by the National Science Foundation (DCB-8316364 and DCB-8710099). Support also provided by the University of Michigan Office of the Vice-President for Research.

\section{References}

Brown, M.B., Karsch, F.J. \& Malpaux, B. (1988) An algorithm to identify changes in hormone patterns. In Interface of Computer Science and Statistics, pp. $779-784$.

Hauger, R.L., Karsch, F.J. \& Foster, D.L. (1977) A new concept for control of the estrous cycle of the ewe based on the temporal relationships between luteinizing hormone, estradiol and progesterone in peripheral serum and evidence that progesterone inhibits tonic LH secretion. Endocrinology 101, 807-817.

Jackson, G.L., Gibson, M. \& Kuehl, D. (1988) Photoperiodic disruption of photorefractoriness in the ewe. Biol. Reprod. 38, 127-134.

Jackson, G.L., Jansen, H. \& Kao, C. (1990) Continuous exposure of Suffolk ewes to a fixed photoperiod disrupts expression of the annual breeding season. Biol. Reprod. 41, 63-73.

Karsch, F.J. \& Foster, D.L. (1975) Sexual differentiation of the mechanism controlling the preovulatory discharge of luteinizing hormone in sheep. Endocrinology 97, 373-379.

Karsch, F.J., Bittman, E.L., Robinson, J.E., Yellon, S.M., Wayne, N.L., Olster, D.H. \& Kaynard, A.H. (1986) Melatonin and photorefractoriness: loss of response to the melatonin signal leads to seasonal reproductive transitions in the ewe. Biol. Reprod. 34, 265-274.

Karsch, F.J., Robinson, J.E., Woodfill, C.J.I. \& Brown, M.B. (1989) Circannual cycles of luteinizing hormone and prolactin secretion in ewes during pro- longed exposure to a fixed photoperiod: evidence for an endogenous reproductive rhythm. Biol. Reprod. 41, 1034-1046.

Legan, S.J. \& Karsch, F.J. (1979) Neuroendocrine regulation of the estrous cycle and seasonal breeding in the ewe. Biol. Reprod. 20, 74-85.

Legan, S.J. \& Karsch, F.J. (1980) Photoperiodic control of seasonal breeding in ewes: modulation of the negative feedback action of estradiol. Biol. Reprod. 23, 1061-1068.

Legan, S.J., Foster, D.L. \& Karsch, F.J. (1977) The endocrine control of seasonal reproductive function in the ewe: a marked change in response to the negative feedback action of estradiol on luteinizing hormone secretion. Endocrinology 101, 818-824.

Malpaux, B., Moenter, S.M., Wayne, N.L., Woodfill, C.J. \& Karsch, F.J. (1988a) Reproductive refractoriness of the ewe to inhibitory photoperiod is not caused by alteration of the circadian secretion of melatonin. Neuroendocrinology 48, 264-270.

Malpaux, B., Robinson, J.E., Brown, M.B. \& Karsch, F.J. (1988b) Importance of changing photoperiod and melatonin secretory pattern in determining the length of the breeding season in the Suffolk ewe. J. Reprod. Fert. 83, 461-470.

Malpaux, B., Robinson, J.E., Wayne, N.L. \& Karsch, F.J. (1989) Regulation of the onset of the breeding season of the ewe: importance of long days and of an endogenous reproductive rhythm. $J$. Endocr. 122, 269-278. 
Niswender, G.D., Midgley, A.R., Jr \& Reichert, L.E., Jr (1968) Radioimmunologic studies with murine, bovine, ovine and porcine luteinizing hormone. In Gonadotropins, pp. 307-312. Ed. E. Rosemberg. Geron-X, Inc., Los Altos.

Niswender, G.D., Reichert, L.E., Jr, Midgley, A.R., Jr \& Nalbandov, A.V. (1969) Radioimmunoassay for bovine and ovine luteinizing hormone. Endocrinology 84, $1166-1173$.

O'Callaghan, D., Roche, J.F., Boland, M.P.\& Karsch, F.J. (1989a) Does a melatonin implant mimic a short-day photoperiodic effect in ewes? J. Anim.Sci.67(Suppl. 1), Abstr. 879

O'Callaghan, D., Roche, J.F., Boland, M.P. \& Karsch, F.J. (1989b) Role of short days in timing the onset and duration of the breeding season in ewes. Biol. Reprod. 40 (Suppl. 1), Abstr. 138.

Poulton, A.L., English, J., Symons, A.M. \& Arendt, J. (1987) Changes in plasma concentrations of LH, FSH and prolactin in ewes receiving melatonin and shortphotoperiod treatments to induce early onset of breeding activity. $J$. Endocr. 112, 103-111.

Robinson, J.E. \& Karsch, F.J. (1987) Photoperiodic history and a changing melatonin pattern can determine the neuroendocrine response of the ewe to daylength. J. Reprod. Fert. 80, 159-165.
Robinson, J.E., Radford, H.M. \& Karsch, F.J. (1985a) Seasonal changes in pulsatile luteinizing hormone secretion in the ewe: relationship of frequency of LH pulses to day length and response to estradiol negative feedback. Biol. Reprod. 33, 324-334.

Robinson, J.E., Wayne, N.L. \& Karsch, F.J. (1985b) Refractoriness to inhibitory day lengths initiates the breeding season of the Suffolk ewe. Biol. Reprod. 32, $1024-1030$.

Thimonier, J., Ravault, J.P. \& Ortavant, R. (1978) Plasma prolactin variations and cyclic ovarian activity in ewes submitted to different light regimens. Annls Biol. anim. Biochim. Biophys. 18, 1229-1235.

Wayne, N.L., Malpaux, B. \& Karsch, F.J. (1990) Photoperiodic requirements for timing onset and duration of the breeding season in the ewe: synchronization of an endogenous rhythm of reproduction. J. comp. Physiol., A166, 835-842.

Worthy, K., Haresign, W., Dodson, S., McLeod, B.J., Foxcroft, G.R. \& Haynes, N.B. (1985) Evidence that the onset of the breeding season in the ewe may be independent of decreasing plasma prolactin concentrations. J. Reprod. Fert.75, 237-246.

Received 16 March 1990 